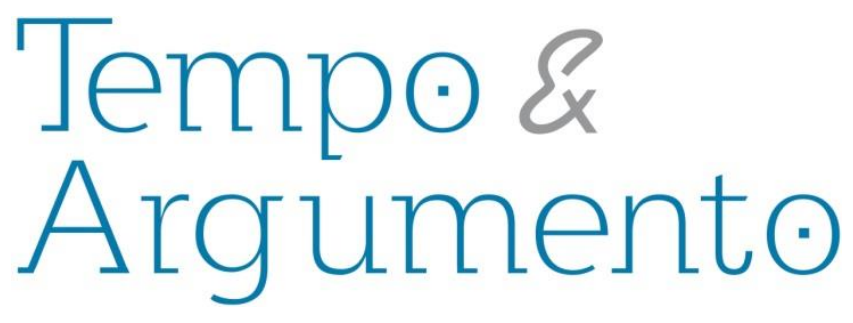

\title{
La izquierda peronista de los años sesenta como fenómeno argentino de la llamada nueva izquierda
}

\begin{abstract}
Resumen
La llamada nueva izquierda fue un fenómeno surgido en los años cincuenta a escala trasnacional, y no son pocas las resonancias que tuvo en Argentina. Sin embargo, aunque desde el campo académico argentino se ha enfocado frecuentemente el estudio de los años sesenta y setenta a través de dicha categoría, no encontramos localmente grupos políticos o experiencias intelectuales de relevancia que hayan asumido como propia esa caracterización del mismo modo a lo sucedido en Europa y Estados Unidos, e incluso en otros países de América Latina. Como categoría analítica aplicable al caso argentino, requiere ser definida de modo tal que las experiencias concretas puedan ser ubicadas dentro o fuera de esa delimitación. Como se verá, la categoría no siempre resulta útil a la hora de definir un sujeto político particular. Estudios sobre expresiones políticas diversas han encontrado similares dificultades a la hora de ubicar a su objeto de estudio dentro o fuera de la llamada nueva izquierda. En caso de utilizar esta categoría de modo analítico, argumentaremos que el fenómeno de la izquierda peronista fue una de sus mayores expresiones locales, aunque no siempre los trabajos académicos sobre el tema coinciden en torno a la relación entre ambas categorías.
\end{abstract}

Palabras clave: Izquierda Peronista. Nueva Izquierda. Años Sesenta y Setenta.

Marxismo. Peronismo.

\section{Para citar este artículo:}

FRIEDEMANN, Sergio. La izquierda peronista de los años sesenta como fenómeno argentino de la llamada nueva izquierda. Tempo e Argumento, Florianópolis, v. 10, n. 24, p. 484 - 509, abr.jun. 2018.

DOI: $10.5965 / 2175180310242018484$

http://dx.doi.org/10.5965/2175180310242018484 


\title{
The Peronist Left of the Sixties as an Argentine Phenomenon of New Left
}

\begin{abstract}
The commonly named New Left was a phenomenon that emerged in the fifties on a transnational scale, and there are no few resonances that took place in Argentina. However, although the Argentine academic field has often focused on the study of the sixties and seventies through that category, we didn't find at a local level political groups or intellectual experiences that have assumed as their own that characterization similarly to what happened in Europe and the United States, or even in other countries in Latin America. As analytical category applicable to the Argentine case, it needs to be defined so that the specific experiences can be placed inside or outside that boundary. We state that the category is not always useful when defining a particular political subject. Studies on various political expressions have found similar difficulties to locate the object of study within or outside the so-called New Left. When using this category in an analytical approach, we will assert that the phenomenon of the left-wing Peronism was one of its greatest local expressions, but not always academic papers on the subject agree about its position within the category.
\end{abstract}

Keywords: Peronist Left. New Left. Sixties and seventies. Marxism. Peronism. 
Ocurre aquí como con los primeros pensamientos de Copérnico. Éste, viendo que no conseguía explicar los movimientos celestes si aceptaba que todo el ejército de estrellas giraba alrededor del espectador, probó si no obtendría mejores resultados haciendo girar al espectador y dejando las estrellas en reposo. Immanuel Kant ${ }^{1}$

\section{Introducción}

El presente trabajo se enmarca en una investigación sobre el impacto que fenómenos de escala trasnacional ejercieron sobre las transformaciones del peronismo en los “largos sesenta". Particularmente, se ocupa del surgimiento y desarrollo de un conjunto de organizaciones políticas, y político-militares, así como espacios y figuras intelectuales, profesionales y del mundo de la cultura, que formaron parte de un entramado complejo y heterogéneo que nominamos como izquierda peronista.

A lo largo del artículo, analizamos la relación entre las categorías de nueva izquierda e izquierda peronista para el estudio de fenómenos vinculados al proceso de radicalización política de los años sesenta y setenta en Argentina. Para ello, se rastrean en primer lugar los primeros usos de la categoría de nueva izquierda en el campo políticointelectual europeo y norteamericano en el contexto de la llamada desestalinización y la revisión trasnacional del marxismo desde mediados de la década del cincuenta. En segundo lugar, pasamos a analizar los usos de la categoría por parte de investigadores argentinos en tres momentos diferentes de la producción bibliográfica sobre el tema. Examinamos las continuidades entre los procesos trasnacionales que dieron lugar a dicha nueva izquierda con el proceso político argentino, problematizando la relación entre izquierda peronista y nueva izquierda. Sobre este punto, señalamos algunas dificultades reconocidas en estudios de caso para ubicar experiencias políticas e intelectuales bajo el

\footnotetext{
${ }^{1}$ Prólogo a la primera edición de Crítica de la Razón Pura, de 1781.
} 
binomio nueva izquierda - izquierda tradicional. En las conclusiones argumentamos, a partir del recorrido realizado, que en caso de utilizar la categoría de nueva izquierda para los años sesenta y setenta argentinos, sería prudente incorporar bajo dicho campo a la izquierda peronista.

Se trata de un estudio exploratorio y cualitativo, que analiza las producciones teóricas y políticas de la llamada nueva izquierda europea y norteamericana, así como los estudios que en nuestro país han retomado dicha categoría para un estudio de fenómenos vinculados a la Izquierda Peronista.

\section{La revisión del marxismo en los años sesenta}

Grupos políticos autoproclamados como nueva izquierda ${ }^{2}$ surgieron a escala trasnacional tras la muerte de Stalin. Acontecimientos como el informe de Nikita Kruschev ante el XX Congreso del Partido Comunista de la Unión Soviética en febrero de 1956, la represión a la revolución húngara y la invasión al Canal de Suez ese mismo año, y el conflicto chino-soviético pocos años más tarde, fueron condiciones para la gestación de dicho fenómeno (HOBSBAWM, 2013; ZOLOV, 2012). En abierta confrontación con el marxismo oficial u ortodoxo sostenido por la URSS, surgieron “ortodoxias rivales" (HOBSBAWM, 2013, p. 355), además de múltiples y variadas renovaciones heterodoxas. En ese marco, diversos espacios políticos comenzaron a autoproclamarse como la nueva izquierda. El primer caso al que accedimos es el de la Nouvelle Gauche francesa, surgida en torno al semanario France Observateur dirigido por Claude Bourdet. Los fundadores de la new left británica, por su lado, afirman haber tomado de allí el nombre para su movimiento (HALL, 2010).

El jamaiquino Stuart Hall, becado en 1951 para irse a estudiar de la colonia a la metrópolis y primer editor jefe de la New Left Review, afirma haber conocido a Bourdet en una conferencia junto con otros impulsores de la nueva izquierda británica. Recuerda que los primeros contactos y discusiones entre el grupo se dieron alrededor de 1954. Sin

\footnotetext{
${ }^{2}$ Colocamos con minúscula y sin comillas el concepto de nueva izquierda, a excepción de aquellos casos en los que se trate de citas textuales en los que respetamos el uso tal como se presenta en las fuentes originales.
} 
embargo, sitúa la fecha de nacimiento de la new left en 1956, a partir de dos sucesos casi simultáneos: “el aplastamiento de la Revolución Húngara por los tanques soviéticos” y "la invasión francesa y británica de la zona del Canal de Suez" (HALL, 2010, p. 163) tras su nacionalización impulsada por Nasser en Egipto. Según el historiador norteamericano Eric Zolov, el informe de Kruschev “dejó atónito al campo socialista” minando el prestigio y la credibilidad internacional de la URSS, constituyendo junto a la invasión de Hungría puntos de inflexión que "rompieron con el apoyo incondicional de muchos participantes de la izquierda al comunismo soviético" (ZOLOV, 2012, p. 5). Eric Hobsbawm indica en un sentido similar que luego de 1956 "una gran mayoría de marxistas se vieron obligados a concluir que los regímenes socialistas existentes (...) estaban lejos de lo que ellos mismos habrían deseado que fuese una sociedad socialista" (HOBSBAWM, 2013, p. 356). La desestalinización condujo, según el historiador, a reconocer que eran necesarias importantes reformas en el campo socialista, y a la vez a "un cierto deshielo intelectual que permitió el replanteamiento (...) de cuestiones firmemente bloqueadas en la era de Stalin". A partir de entonces, ya no iba a haber "un único movimiento comunista internacional, monolítico y monocéntrico", en referencia a la perspectiva hegemónica de la URSS (y en función de lo cual la oposición del trotskismo constituyó un antecedente importante para la nueva izquierda), sino que en el centro de los debates marxistas aparecería una variedad de "vías nacionales hacia el socialismo" (p. 355). Las afirmaciones de Hobsbawm pueden leerse en su doble carácter: el del historiador del siglo XX, pero a la vez el del testigo. Era él uno de los que habían impulsado a mediados de los cincuenta esta nueva corriente.

En efecto, tras los sucesos de 1956 se conformaron en Gran Bretaña dos grupos que editaron dos revistas: The New Reasoner — por Edward P. Thompson y John Savilley Universities and Left Review — por Stuart Hall, Charles Taylor, Raphael Samuel y Gabriel Pearson. Eric Hobsbawm fue uno de los intelectuales marxistas que escribieron en ambas publicaciones. Comenzaron a editarse casi simultáneamente en 1957 con el propósito de conformar una "nueva izquierda" y rechazando las tendencias dominantes en los partidos laborista y comunista. Finalmente, en 1960 se fusionaron ambos consejos 
editoriales para conformar la New Left Review. ${ }^{3}$ Como empezamos a ver, nueva izquierda es una categoría nativa acuñada por académicos y universitarios europeos bajo el influjo existencialista del “compromiso" de los intelectuales (GILMAN, 2012; TERÁN, 1991).

En el primer número de Universities and Left Review un artículo del propio Bourdet daba cuenta de lo que llamó "un nuevo fenómeno: la aparición de una tercera corriente socialista: la Nueva Izquierda". ${ }^{4}$ Según el inspirador de la new left británica, en Francia fue un nombre que sirvió para nombrar a tres grupos diferentes: en primer lugar, la propia Nouvelle Gauche, liderada por él y formada por ex comunistas y socialistas; en segundo lugar, Jeune Republique, un grupo de socialistas cristianos; y en tercer lugar, el Mouvement de Liberation du Peuple, espacio conformado centralmente por jóvenes de clase trabajadora con un fuerte contenido socialista y críticos del marxismo ortodoxo. ${ }^{5}$ Los tres grupos eran antiestalinistas, anticolonialistas y anticapitalistas. La novedosa apropiación del marxismo habilitaba a la confluencia de tradiciones políticas, religiosas e intelectuales diversas: nacionalismo, cristianismo, socialismo y comunismo.

Lo que entusiasmó a los ingleses de la experiencia francesa, según Hall, consistía en el hecho de asumir una "tercera posición", tanto frente a las tendencias existentes en las izquierdas (el estalinismo y la socialdemocracia, sin hacer mención al trotskismo), como también frente a la disputa entre Estados Unidos y la Unión Soviética por su influencia en Europa. Los efectos del Estado de bienestar keynesiano habilitaban a pensar en una "sociedad «poscapitalista»" (HALL, 2010, p. 164). Se asomaba una relectura de la experiencia laborista y los posibles vínculos con un porvenir socialista. En forma llamativamente similar al caso argentino, donde intelectuales de izquierda aportaron a una relectura del fenómeno peronista (ALTAMIRANO, 1992; SIGAL, 1991; TERÁN, 1991), Hall justifica de este modo el vínculo de la nueva izquierda con el laborismo inglés.

\footnotetext{
3 New Left Review, Breve historia de la New Left Review, s/f, URL: http://newleftreview.es/history._Las colecciones completas de ambas revistas están disponibles en http://banmarchive.org.uk/archive_index.htm. Puede consultarse, por ejemplo, el artículo de Edward Thompson titulado "The new left”, en el número 9 de New Reasoner, 1959.

${ }^{4}$ Bourdet, Claude, “The French Left", Universities and Left Review, 1(1), 13-17, 1957.

${ }^{5}$ Según Bourdet, los tres grupos tenían muchos puntos en común, actuaban unificados en muchos casos y aspiraban en 1957 a unificarse en un único partido. Ibidem, p. 16.
} 
Reconocíamos que la suerte del socialismo en Gran Bretaña estaba inexorablemente unida al destino y a las fortunas del laborismo. Reconocíamos que para bien o para mal, el Partido Laborista era el partido que había dirigido a la amplia mayoría de la clase trabajadora con una política reformista. Honrábamos su vínculo histórico con el movimiento sindical. Lo reconocíamos como el motor de la revolución del «Estado de bienestar» de 1945. (HALL, 2010, p. 176-177)

Luego destaca la necesidad de "superar la división tradicional entre los intelectuales y la clase trabajadora" (p. 178) y "que el proyecto socialista tenía que estar enraizado en el aquí y ahora y conectar con la experiencia viva, con lo que desde entonces se ha dado en llamar «lo nacional-popular»", o "populista en el sentido que daban los narodnik a «ir al pueblo»” (p. 179). Con un tono algo más autocrítico, Hobsbawm asume que el intento de "unirse al pueblo" por parte de estos intelectuales radicalizados era expresión de una falta: su origen de clase estaba bien alejado de obreros y campesinos. ${ }^{6}$

La propuesta inglesa repercutió también en los Estados Unidos. Charles Wright Mills escribió en 1960 su "carta a la nueva izquierda"” con motivo de la publicación del libro Out of apathy coordinado por E. Thompson (1960) y editado por la New Left Review. Leído por Ernesto Guevara en Bolivia, Wright Mills era junto con Huberman, Sweezy y Baran un representante de la new left estadounidense, conformada a la vez al calor de la revolución cubana (KOHAN, 2011). ${ }^{8}$

También en México, donde los intercambios con Estados Unidos eran muy fluidos, el concepto de nueva izquierda comenzó adquirir fuerza política (ZOLOV, 2012). Pero no es el propósito continuar aquí analizando itinerarios de la llamada nueva izquierda en otros países a lo largo de la década, sino dar cuenta de sus primeros usos, previos al movimiento mundial del '68. Es cierto que a partir del mayo francés comenzó a ser utilizada como categoría analítica para dar cuenta de ese vasto fenómeno de manifestaciones estudiantiles con epicentro en París. Hoy en día se sigue utilizando de modo análogo en los trabajos sobre las décadas del sesenta y setenta, mientras actores

\footnotetext{
${ }^{6}$ Lo cual podía llevar a asumir un lugar de vanguardia intelectualizada que la mantendría alejada de la clase trabajadora. Una autocrítica similar estaría presente en algunos sectores de la izquierda peronista universitaria. "De base y con Perón. Un documento autocrítico de las ex - cátedras nacionales", Antropología zer mundo 4/10, pp. 27-34, junio 1972.

7 Wright Mills, Charles, “Letter to the New Left", New Left Review, 5, 1960.

${ }^{8}$ Según reconstruye este autor, cuando Wright Mills murió en 1962 estaba trabajando en torno a un libro titulado The new left.
} 
políticos contemporáneos vuelven una y otra vez sobre la idea de una refundación de la tradición socialista. Es decir, que si nació como una categoría a la vez analítica y política en tanto fue acuñada por intelectuales-militantes (asociado a la idea sartreana del “intelectual comprometido” o la gramsciana del “intelectual orgánico”), hoy día es utilizada tanto por trabajos académicos sobre las décadas del '60 y '70, como por actores políticos diversos, ubicándose estos dos usos en espacios claramente separados.

Desde el punto de vista teórico e ideológico, la nueva izquierda trasnacional de los sesenta fue un espacio intelectual heterogéneo con intenciones de articulación política casi nunca logradas. Pero había elementos distintivos. La oposición al dogmatismo y el determinismo económico de la ortodoxia soviética impulsó la revisión del marxismo, junto con el hallazgo de los Manuscritos económico-filosóficos de Marx de 1844, disponibles a partir de esos años. ${ }^{9}$ El llamado marxismo humanista que impulsaron autores como Jean Paul Sartre, Erich Fromm, Raya Dunayevskaya, entre muchísimos otros $^{10}$, fue una de las principales corrientes de la época (PONZA, 2006; TERÁN, 1991). Igualmente relevante para el porvenir intelectual de la nueva izquierda fue la posterior reacción contra ese humanismo de parte de Louis Althusser con la reivindicación de un marxismo científico. ${ }^{11}$ Los humanistas rescataron algunos aspectos de la filosofía hegeliana, los escritos del joven Marx -sobre todo el concepto de enajenación- y los cuadernos de la cárcel de Gramsci, entre otros textos que no formaban parte de los principales estantes de la biblioteca soviética. Althusser sostuvo que el joven Marx no tenía mucho que ver con el autor de El Capital, mientras los humanistas señalaron las continuidades por sobre las rupturas. El debate intelectual de izquierdas vivía un “renacimiento", del cual lo aquí esbozado constituye tan solo una aproximación mínima.

La revisión y renovación del marxismo fue un fenómeno mundial, pero la categoría de nueva izquierda comenzó a cuadrar en todos aquellos grupos políticos y experiencias

\footnotetext{
${ }^{9}$ En español los manuscritos de Marx fueron incluidos por Fondo de Cultura Económica en 1962 en un libro de Erich Fromm titulado Marx y el concepto de hombre. Según Ponza, el semanario Primera Plana lo incluyó en una lista de best-sellers a principios de 1963. (PONZA, 2006).

${ }^{10}$ Marxists Humanism and the "new left", URL: http://www.marxists.org/subject/humanism/index.htm

${ }^{11}$ Sobre la mirada anti-humanista de Louis Althusser el lector puede recurrir a su "Marxismo y Humanismo", en La revolución teórica de Marx, México, Ed. Siglo XXI, 2011. El texto fue escrito en francés en 1965 y publicado en español dos años más tarde. Sobre el impacto de Louis Althusser sobre la Nueva Izquierda véase el trabajo de Sebastián Gómez (2016).
} 
de las izquierdas que no adherían a la ortodoxia, ya sea porque las particularidades de cada país requerían una seria revisión de la "cuestión nacional”", o simplemente porque los partidos tradicionales de izquierda sufrían rupturas en función de críticas internas que no encontraban el modo de encauzarse sin fisuras. Pero sin dudas que una de los principales impulsos para este fenómeno fue la caída de la hegemonía soviética dentro del mundo intelectual de las izquierdas, en un contexto en el que la heredera de la revolución de octubre se constituyó en una de las dos superpotencias en un mundo bipolar en plena guerra fría, y no iba a vacilar en ampliar su esfera de acción con metodologías imperialistas. Las revueltas obreras y estudiantiles de la década se sucedían más allá de la influencia soviética, que en muchos casos se ocupó de reprimirlas. Fue tal el grado de internacionalización del fenómeno del '68, que cruzó dos conjuntos de fronteras globales: aquella que separaba a países centrales de países dependientes, pero también la que distinguía entre países socialistas y capitalistas.

\section{Peronismo y Nueva Izquierda en la bibliografía académica sobre el caso argentino}

Son numerosos los trabajos que han enfocado el estudio del período a través de la relación entre peronismo e izquierdas, el fenómeno de la radicalización política y el surgimiento de una nueva izquierda (ALTAMIRANO, 1992; GEORGIEFF, 2009; HILB; LUTZKY, 1984; TERÁN, 1991; TORTTI, 2007). Sin embargo, y a diferencia de lo sucedido en Europa y Estados Unidos, e incluso en otros países de América Latina, no encontramos en nuestro país organizaciones que se autoproclamen como nueva izquierda durante este período. A pesar de ello, desde la historia intelectual y la historia reciente se ha utilizado frecuentemente esa categoría. Proponemos explorar esos usos para indagar acerca de la relación entre nueva izquierda e izquierda peronista en la bibliografía académica.

Entre quienes abordan los sesenta delimitando una nueva izquierda para el caso argentino, se pueden distinguir tres tendencias principales que surgen en tres etapas diferentes de la producción de conocimiento sobre el tema. Primero, como lo hacen Hilb

\footnotetext{
${ }^{12}$ Por ejemplo, en torno a la cuestión del "neocolonialismo" en lo que se empezó a llamar como "Tercer Mundo", fenómeno que requería un nuevo tipo de respuestas.
} 
y Lutzky (1984) en una publicación pionera, están los estudios focalizados en las experiencias políticas revolucionarias que optaron por la lucha armada, poniendo en primer lugar el asunto de la violencia política durante los sesenta y setenta como característica a considerar. Se trataría de una nueva izquierda político-revolucionaria. Una segunda generación de trabajos ha privilegiado el análisis de la "nueva izquierda intelectual" haciendo foco en la producción teórica y el trabajo editorial de una serie de figuras o grupos de la cultura y las ciencias sociales durante la década del sesenta (ALTAMIRANO, 1992; TERÁN, 1991). En tercer lugar, y nutriéndose sobre todo de éstos últimos trabajos, producciones algo más recientes que hacen foco en las experiencias partidarias, políticas e intelectuales que dieron lugar a la emergencia de esa nueva izquierda, más allá de la opción por las armas que hayan privilegiado algunas de ellas (PUCCIARELLI, 1999; TARCUS, 2007; TORTTI, 1999, 2002, 2007). A continuación examinaremos cuál es el lugar que ocupa la izquierda peronista según los estudios mencionados.

Claudia Hilb se propone analizar "las condiciones de surgimiento y desarrollo de las concepciones de los grupos que hemos agrupado bajo la denominación de «Nueva Izquierda» de los años 60" (1984, p. 11). La autora definirá esa nueva izquierda en función de la aceptación de la violencia y la guerra como únicas vías para acceder al poder. Quedarán incluidas en esa definición las organizaciones armadas peronistas y no peronistas.

Oscar Terán, en cambio, hará una historia de las ideas del período 1956-1966, limitándose a determinadas figuras intelectuales, como Silvio Frondizi, Hernández Arregui, Jorge A. Ramos, Juan J. Sebreli, Ismael Viñas, Oscar Masotta, entre otros, y también a revistas, como Contorno, Pasado y Presente, La Rosa Blindada, Cuestiones de Filosofía, entre otras. La característica definitoria de la nueva izquierda no será los modos de justificar la violencia política, sino la diferenciación respecto del esquematismo teórico de la izquierda tradicional y la relectura realizada por muchos intelectuales respecto del peronismo. El peronismo aparece como un afluente de la nueva izquierda en términos de “nacionalistas de izquierda”, pero será sobre todo la ruptura con el estalinismo y la 
incorporación de enfoques marxistas más heterodoxos (Sobre todo Gramsci y Sartre) la que dará nacimiento a esa “nueva izquierda argentina” (TERÁN, 1991, p. 103).

Carlos Altamirano (1992), por su parte, describe una “situación revisionista” (p. 8) a partir de 1955, que provendría de la izquierda, ya sea al margen de los partidos tradicionales, o también en sus márgenes, generando rupturas partidarias. El autor sostiene que la reinterpretación del peronismo iría acompañada de una resignificación más general de los postulados y premisas de la izquierda y de una fuerte crítica a los errores cometidos en el pasado. Sobre todo, resalta el papel asumido por el Partido Comunista Argentino y por el Partido Socialista al caracterizar al primer gobierno peronista como fascista. Si la "izquierda tradicional" no estaba dispuesta a asumir esos errores, la salida habría sido el surgimiento de la “«nueva izquierda»” (p. 24). Afirma el autor que el peronismo se reinterpretó a sí mismo al incorporar elementos de la teoría marxista. En esa relectura jugaron un rol clave, según Altamirano, Rodolfo Puiggrós, Abelardo Ramos y en menor medida Hernández Arregui y John William Cooke (p. 9). Resultado de esas mutaciones surgiría un "nacionalismo de izquierda” (p. 38).

Quien no ha utilizado la categoría de nueva izquierda fue Silvia Sigal (1991). En su trabajo, narra a posteriori, propuso "reconstruir trozos significativos del discurso y comportamiento de los intelectuales" (SIGAL; TERÁN, 1992). A la hora de analizar el fenómeno intelectual de los sesenta, se refirió a la "intelectualidad crítica" o "marxista", a "las izquierdas", o más específicamente a los diferentes espacios que identifica, como la "izquierda nacional”, "grupos trotskistas", el "progresismo" o el "nacionalismo". Solo eventualmente menciona las "nuevas corrientes de la izquierda” y en plural, por lo que no le otorga un estatus conceptual homogeneizador a todas ellas.

Si para Hilb, Terán y Altamirano, no había demasiadas dudas acerca que dentro de lo que decidieron llamar nueva izquierda era significativa la presencia de individuos y grupos de identidad peronista, creemos que es en una segunda generación de trabajos sobre el tema, desde finales de la década del '9o hasta la actualidad, que la pertenencia de parte del peronismo a la nueva izquierda comenzó a ser puesta en duda o interpretada en forma ambigua por los estudiosos del período. 
En una importante compilación de trabajos sobre el tema, Alfredo Pucciarelli define a la nueva izquierda como a un

complejo y expansivo conglomerado de fuerzas sociales y políticas que, a pesar de no haber generado un actor político unificado, encabezó un vasto proceso de protesta social, confrontación ideológica y activación política, hacia fines de la década del sesenta. Un haz de fuerzas que, portadoras de programas que combinaban cuestiones tales como "liberación nacional", "socialismo" o "revolución", imprimieron, en la sociedad argentina, los impulsos de una nueva etapa de contestación generalizada. Un lenguaje compartido y un común estilo político que daban cierta unidad "de hecho" a grupos sociales, generacionales y herederos de diversas tradiciones políticas e ideológicas: peronismo, izquierda tradicional, nacionalismo y grupos católicos influenciados por la “teología de la liberación” (PUCCIARELLI, 1999, p. 15).

La nueva izquierda estaría conformada según el autor por fuerzas sociales y políticas de izquierda que habían heredado diversas tradiciones entre las que se incluye la izquierda tradicional pero también el peronismo, nacionalismo y catolicismo. Cierta unidad ("lenguaje compartido", “estilo político") en la diversidad ("complejo conglomerado", “diversas tradiciones") es la que parece visualizar Pucciarelli en ese espacio político que encabezó el ciclo de protestas a finales de los sesenta. Se puede sostener que la nueva izquierda comienza a ser pensada como una amplia red de relaciones donde el vínculo tejido entre sus elementos (guerrillas, partidos, agrupaciones, revistas, intelectuales, etc.) va a ser de grado muy variable.

Un enfoque similar es el de M. Cristina Tortti, que define la "nueva oposición" y la “nueva izquierda" como "una heterogénea y potente fuerza renovadora cuyo despliegue permite visualizarla como movimiento social a la vez que como actor político" (TORTTI, 1999, p. 222). En este caso la nueva izquierda es un actor político, pero reaparece la figura de lo heterogéneo. La idea de "nueva oposición” se explica teniendo en cuenta que lo que unificaba a ese espacio diverso — cuyo exponencial crecimiento sucedió después del Cordobazo — era la oposición a la dictadura militar (1966-1973).

¿Están contenidas las organizaciones de la izquierda peronista en lo que estos autores definen como nueva izquierda? 
Pucciarelli describe una serie de procesos de los que difícilmente puede dejarse afuera a los grupos peronistas. Se refiere a "las protestas sociales", el "intenso debate ideológico en las universidades", la "radicalización del movimiento estudiantil”, los cuestionamientos al "sentido (...) de las prácticas profesionales" y a las autoridades institucionales, entre otros “discursos y acciones" producidas por la nueva izquierda en el contexto del Gran Acuerdo Nacional (GAN) que tuvo como desenlace el retorno del peronismo al gobierno (1999, p. 5). Como documenta otro estudio del mismo volumen, en 1971 “el avance arrollador del peronismo ya era fácilmente perceptible” (DE AMÉZOLA, 1999, p. 108). Pero Pucciarelli afirma luego que después del Cordobazo la presencia de la nueva izquierda fue tan determinante que se le debe otorgar "la misma envergadura que la de los dos contendientes principales, Peronismo y Fuerzas Armadas" (p. 16). Aquí entonces, peronismo y nueva izquierda aparecen en campos separados. Ésta última, propone, debe ser considerada un "«tercero incluido" en un escenario que (...) ha sido presentado como excesivamente dominado por la confrontación entre la guerrilla, las Fuerzas Armadas y el Movimiento Peronista" (p. 20).

Tortti incluye en principio a la izquierda peronista dentro de la nueva izquierda. Se trataba, según ella, de una “doble pertenencia” en tanto la izquierda peronista "formaba parte, simultáneamente, de otro campo político que se unificaba en el reconocimiento del liderazgo de Perón". Pero la autora también afirma, en un sentido muy similar al de Pucciarelli, que la nueva izquierda "estuvo a la vanguardia de la lucha contra la dictadura", sin poder constituir "una alternativa política al peronismo y a las organizaciones armadas", por lo que "fue quedando afuera" cuando se abrió el juego electoral (p. 225).

Si “esta «nueva oposición» o «nueva izquierda», se volvió particularmente amenazante a partir del '69 y del crecimiento de la guerrilla durante los setenta", la izquierda peronista no puede suponerse entonces como elemento exterior a la nueva izquierda. Esto es especialmente cierto para la organización Montoneros que se transformó en la más importante de las organizaciones guerrilleras y en un espacio hegemónico dentro de la izquierda peronista. En otras palabras: si el peronismo no está incluido en la nueva izquierda, es dudoso que ésta haya estado "a la vanguardia" de la lucha contra la dictadura. Pero si las fracciones de izquierda del peronismo sí formaban 
parte de ella, tenemos entonces un gran sector de la nueva izquierda que no quedó fuera del juego de opciones políticas hacia 1973. Lo que sucedió, en cambio, fue que la heterogeneidad de la nueva izquierda se hizo valer cuando la dictadura llegó a su fin. A la nueva izquierda le faltaba, para ser sujeto, ser parte de un proceso de autoidentificación, más allá de la resistencia a la dictadura. La izquierda peronista aceptó tácticamente la participación electoral, sin dejar de afirmar la necesidad estratégica de una "guerra popular revolucionaria" aun después del acceso al gobierno por parte del peronismo, lo que ella consideraba un asunto diferente de la toma del poder "definitiva" por parte del pueblo (GONZÁLEZ CANOSA, 2018). En otras palabras, debería plantearse como problema hasta qué punto solo la oposición a la dictadura unificaba a espacios políticos que, si bien compartían también el ideario del socialismo, no iban a actuar como sujeto político colectivo ante la posibilidad siempre presente que las urnas sean desempolvadas.

\section{De la nueva izquierda a la izquierda peronista}

Si bien los estudios locales sobre el tema en pocas oportunidades se refieren a la existencia pionera de una autodenominada nueva izquierda francesa, británica y norteamericana, sí acuden al contexto internacional para dar cuenta de ese vasto fenómeno que indudablemente fue de alcance global. No son pocos los fenómenos vinculados a la llamada nueva izquierda trasnacional que tuvieron resonancia en nuestro país: fracturas en los partidos tradicionales de la izquierda, experiencias intelectuales de contenido socialista y conformación de nuevos grupos políticos alrededor de ellas, confluencia del marxismo con otras tradiciones de pensamiento como el nacionalismo, el humanismo, el existencialismo y el catolicismo, el surgimiento de un cristianismo revolucionario, las revueltas estudiantiles y una fuerte ruptura generacional. No es posible negar que variantes argentinas de esas experiencias confluyeron alrededor de la conformación de la izquierda peronista, aunque no exclusivamente en torno a ella. ${ }^{13}$

\footnotetext{
13 Téngase en cuenta, por ejemplo, la articulación de marxismo y nacionalismo en revistas como Columnas del Nacionalismo Marxista editada en 1957, en la obra de Rodolfo Puiggrós y su propuesta de un nacionalismo popular revolucionario (ACHA, 2006; FRIEDEMANN, 2014), y en general en las experiencias políticas e intelectuales de la izquierda nacional y la izquierda peronista. De equivalente importancia resultó la articulación de un cristianismo revolucionario con la identidad peronista en revistas como Cristianismo y Revolución y la organización montoneros, entre otros grupos (CAMPOS, 2016; DONATELLO, 2010; MORELLO, 2007).
} 
Los usos locales de la categoría de nueva izquierda se deben a una conceptualización analítica y no nativa. De igual modo utilizamos la categoría de izquierda peronista: como categoría analítica y relacional. Llamamos izquierda peronista a una zona político-intelectual de múltiples manifestaciones que, conservando cierta heterogeneidad, formaban parte de la cultura política de izquierdas, incorporando categorías y horizontes propios del socialismo y la tradición marxista mientras asumían su pertenencia o adhesión al movimiento peronista. Así, el regreso de Perón al país y del peronismo al gobierno, desde la perspectiva de los actores, podía constituir una etapa o un momento en la construcción del socialismo. La categoría escogida permite distinguir a figuras y grupos que articularon marxismo y peronismo de aquellos que siendo peronistas no asumieron el propósito del socialismo ni hicieron suyos los aportes del marxismo.

Por otro lado, la utilización de una categoría analítica definida por el investigador nos evita la decisión tendenciosa de escoger entre nominaciones que, utilizadas por los actores, expresan matices político-ideológicos. Nos referimos, por ejemplo, a la idea de “peronismo revolucionario" acuñada entre otros por John William Cooke ${ }^{14}$ y Bernardo Alberte $^{15}$, y heredada por diversas organizaciones de la izquierda peronista, la de “nacionalismo popular revolucionario" difundida por Rodolfo Puiggrós ${ }^{16}$ o la de “tendencia revolucionaria del peronismo". ${ }^{17}$ En definitiva, entendemos a la izquierda peronista de los años sesenta y setenta argentinos como un objeto particular de la historia de las izquierdas así como de la historia del peronismo (ACHA, 2009), pero también como un producto local de una serie de fenómenos políticos e ideológicos de escala trasnacional.

Retomando el derrotero global del marxismo, éste se renovó a mediados de los cincuenta y tuvo su apogeo durante los años sesenta. Despojado de su carácter dogmático-doctrinario (HOBSBAWM, 2013; TARCUS, 2013), ahora podía relacionarse con

\footnotetext{
${ }^{14}$ Cooke, J.W., 2014. Peronismo y Revolución. Obras Completas. Tomo V, Buenos Aires: Colihue.

${ }^{15}$ Por ejemplo, la revista Con Todo editada por el Mayor Bernardo Alberte en 1969 se subtitula "órgano del peronismo revolucionario".

${ }^{16}$ Puiggrós, R., 1967. Las izquierdas y el problema nacional. Buenos Aires: Ed. Jorge Álvarez.

${ }^{17}$ Utilizada desde finales de los sesenta, aunque mayormente en el contexto del regreso del peronismo al gobierno entre 1973 y 1975. Era una categoría de uso corriente en los medios de prensa. Véase por ejemplo: "Sobre la universidad habló el Lic. Villanueva”, La Nación, 14 de febrero de 1974, p. 7; “Los errores de la tendencia", La Nación, 5 de enero de 1975, p. 8
} 
otras corrientes de pensamiento, con otras cosmovisiones. ${ }^{18}$ Como observa Hobsbawm, muchos movimientos de liberación nacional de Asia, África y Latinoamérica, se sintieron atraídos por consignas marxistas y se plantearon el objetivo del socialismo, aunque "a menudo unido a un adjetivo, como socialismo africano, socialismo islámico, etc." (HOBSBAWM, 2013, p. 357). En el caso que estudiamos, el adjetivo que asumió el peronismo dio lugar a la idea de un "socialismo nacional", lo cual alimentó expectativas en la izquierda peronista. Esto se puede ver, por ejemplo, en el caso de Rodolfo Puiggrós, quien mantuvo una correspondencia con Perón que lo mostraba ocupado por establecer vínculos con la juventud universitaria:

Al declarar Usted que "el peronismo es el socialismo nacional" nos ha dado un arma de extraordinaria eficacia para destruir la influencia que queda de las sectas antinacionales y para abrir la perspectiva de los cambios sociales que la Patria exige. ${ }^{19}$

Es en la variación de escalas (REVEL, 2005), desde lo trasnacional a lo local, pasando por lo regional, que puede problematizarse la categoría de nueva izquierda. Por cierto, si el fenómeno de la nueva izquierda de los sesenta trascendió a las fronteras nacionales, los trabajos de investigación abocados a un objeto más localizado geográficamente no pueden más que tomar dicho fenómeno como una variable explicativa sin perder de vista particularidades localmente situadas. La categoría de nueva izquierda puede resultar útil para una contextualización trasnacional, teniendo en cuenta que fue en los países centrales que sus primeros usos tuvieron lugar. Su traslado para el estudio de experiencias regionales o nacionales puede estar justificado como construcción analítica del objeto, entre otras delimitaciones viables. En ese sentido,

\footnotetext{
18 “Precisamente cuando el marxismo está fuertemente presente en la escena intelectual es más difícil de mantener la rígida y mutuamente excluyente separación de las ideas marxistas y no marxistas, puesto que marxismo y no marxismo funcionan en un universo cultural que los contiene a ambos. Así pues, en la década de 1960, la tendencia en sectores de la izquierda a combinar a Marx con el estructuralismo, con el psicoanálisis, econometrías académicas, etc., proporciona, entre otras cosas, la prueba del fuerte atractivo del marxismo en los intelectuales de la universidad en aquellos tiempos". (HOBSBAWM, 2013, p. 244).

${ }^{19}$ Rodolfo Puiggrós a Juan D. Perón. Buenos Aires, 4 de agosto de 1971. Juan Domingo Perón Papers, Box 5 , Hoover Institution Archives, Stanford University. Sobre otras repercusiones de la idea de socialismo nacional en la izquierda peronista, pueden mencionarse las "Mesas Redondas sobre Socialismo Nacional" organizadas por los Centros Iberoamericanos para la Emancipación Nacional, espacio impulsado entre otros por Hernández Arregui y Carpani. Luis Fernando Calviño papers. Box 1. Hoover Institution Archives, Stanford University
} 
argumentamos que el fenómeno de la izquierda peronista fue una de las expresiones, para el estudio del caso argentino, de lo que la bibliografía denomina nueva izquierda.

El auge de un marxismo revisado fue un fenómeno mundial pero el peronismo fue un movimiento específicamente nacional que supo ser emparentado con los llamados movimientos de liberación nacional entroncados con la idea de "tercer mundo" (MANZANO, 2014). Si la “desperonización” proyectada por la autodenominada "revolución libertadora" fue un rotundo fracaso (DE RIZ, 2000), el resultado fue lo que algunos autores han denominado una "peronización" (BARLETTA, 2000; CHAMA, 2006; DIP, 2017; FRIEDEMANN, 2017) o al menos un "revisionismo" (ALTAMIRANO, 1992), especialmente de las capas medias, profesionales, estudiantes e intelectuales que durante el primer peronismo habían ejercido una fuerte oposición. La confluencia entre la identidad peronista y elementos de la tradición marxista fue parte del doble proceso de peronización e izquierdización de la sociedad, aunque existen antecedentes de lo que podría considerarse una alianza entre ideas nacionalistas-populares y perspectivas deudoras de la tradición marxista durante el primer peronismo e incluso antes (FRIEDEMANN, 2014; HERRERA, 2016).

Tras el golpe de Estado a Perón en 1955 dicha confluencia comenzó a ser más visible y a hacerse presente en la vida política, y con más potencia una vez avanzada la década del sesenta. Una nueva generación se apropió de los símbolos peronistas y emparentó sus ideales revolucionarios con el regreso de Perón, que alimentó desde el exilio esa mirada sobre sí mismo. En ese período, el encuentro entre ambas tradiciones políticas se fortaleció tanto en la militancia como en la construcción conceptual por parte de sus referentes teóricos. Hernández Arregui, Puiggrós, Ramos, Cooke, Carri, Walsh e incluso Jauretche, entre otros, dejaron que el peronismo se nutriera cada vez más de categorías de análisis marxistas. Casi todos ellos fueron más allá, y se dejaron atrapar por la posibilidad de que ese movimiento político fuera una herramienta política para la construcción del socialismo. 


\section{Límites de la categoría de nueva izquierda}

Se puede sostener que se trata de una categoría analítica utilizada por muchos estudiosos del caso argentino, pero cuyo nacimiento está relacionado con la acción política-intelectual europea y norteamericana. Seguramente haya ejercido su impacto que el primer estudio teórico acerca del tema hecho por investigadores argentinos se haya realizado en el marco de la academia francesa durante el exilio a comienzos de los ochenta (HILB; LUTZKY, 1984), siendo justamente en Francia donde la categoría de nueva izquierda resultó precursora en el campo de lo político, utilizada por intelectuales que a la vez eran militantes. Por otro lado, Oscar Terán, autor de un trabajo ya canónico sobre la temática, estuvo exiliado en México ${ }^{20}$, donde el movimiento de la nueva izquierda surgió fruto de un intercambio muy fluido con Estados Unidos. ${ }^{21}$ El subtítulo elegido por el intelectual argentino ("la formación de la nueva izquierda intelectual en la Argentina 1956-1966") no puede menos que haber incidido en las categorizaciones realizadas por trabajos posteriores. Tanto en Europa como en Estados Unidos e incluso en México ${ }^{22}$, la idea de nueva izquierda fue impulsada por grupos de intelectuales marxistas o estudiantes universitarios que frecuentemente publicaban revistas, conformaron grupos que llevaron ese nombre o reivindicaban su uso, y que nacían con el propósito de la intervención política.

En el caso de la Argentina, en cambio, ninguna de las revisitadas "revistas de la Nueva Izquierda”23 utilizó esa denominación y no hemos hallado grupo político de relevancia que asumiera esa caracterización. ${ }^{24}$

\footnotetext{
${ }^{20}$ Terán, op cit. Para la trayectoria intelectual de Terán, ver Carli (2013).

${ }^{21}$ Zolov, op cit.

${ }^{22}$ Véase por ejemplo el ejemplar de la revista nueva izquierda editada por estudiantes universitarios en http://larotativa.nexos.com.mx/wp-content/uploads/2014/05/001-copia.jpg

${ }^{23}$ Muchos trabajos académicos estudian las "revistas de la nueva izquierda" de los años sesenta. Han sido incluidas bajo esa denominación Pasado y Presente, La Rosa Blindada, Contorno, Che, Situación, Cristianismo y Revolución, Los libros, Envido, Antropología zer Mundo, entre otras. Sin ser exhaustivos, mencionamos algunas indagaciones en torno a esas revistas que parten del presupuesto de ubicarlas como expresiones de la nueva izquierda, aunque no en todas ellas el concepto es problematizado. El propio Terán (1991) analiza Contorno, Pasado y Presente, La Rosa Blindada y Cuestiones de Filosofía, entre otras. Pablo Ponza (2006) se refiere también a Contorno, Revista de la Universidad de Buenos Aires (RUBA) y Cuestiones de Filosofía. Un trabajo mucho más profundo sobre Pasado y Presente puede encontrarse en Burgos (2004). Sobre Los libros y Cristianismo y Revolución, especialmente en su posicionamiento frente al Cordobazo, véase Celentano (2014). Sobre la revista Che, Tortti (2002) , y acerca de Antropología zer Mundo, Barletta y Lenci (2000).
} 
Si bien es cierto que la idea de una renovación de las izquierdas estaba presente en los debates de la época, el uso de la categoría de nueva izquierda en Argentina por parte de los actores parece haber sido minoritario, y en todo caso, asumido con un tono despectivo. Tanto Terán como Altamirano mencionan en sus trabajos que el Partido Comunista Argentino le dedicó un número de sus Cuadernos de Cultura en 1960 a cuestionar a la “nueva izquierda” (ALTAMIRANO, 1992, p. 11; TERÁN, 1991, p. 107). ${ }^{25}$ Pero la cita es inexacta. La publicación partidaria dirigida por Héctor Agosti se refirió centralmente a la "neoizquierda”, en la pluma de Juan Carlos Portantiero, Héctor Agosti y Ernesto Giudice, entre otros. Este último utiliza también las categorías de "neosocialismo y neomarxismo", y emparenta la "neoizquierda" con la "izquierda nacional”. ${ }^{26}$

La publicación generó la respuesta de quienes eran ubicados bajo ese rótulo por el comunismo argentino, como Jorge Abelardo Ramos, para quien la "izquierda nacional” no tenía nada de nuevo, o por Juan José Sebreli que respondía en El escarabajo de oro que las críticas de esa publicación eran "generales y abstractas" y estaban dirigidas a "grupos imprecisos y vagos", como la nueva izquierda, la heterodoxia, la izquierda nacional, los escritores «comprometidos», los intelectuales sin partido, entre otros (SIGAL, 1991).

${ }^{24}$ En el Diccionario biográfico de la izquierda argentina. De los anarquistas a la "nueva izquierda" 1870-1976), dirigido por Horacio Tarcus (2007), la lista de siglas utilizadas durante toda la obra da cuenta de la gran variedad de organizaciones de izquierda existentes en la historia política de nuestro país. En ningún caso se utiliza el nombre de nueva izquierda que sí está incluido en el título del diccionario. Tampoco existen espacios políticos ni revistas que se denominen o reivindiquen literalmente una nueva izquierda, con dos excepciones: 1) un boletín interno del grupo trotskista "El Proletario", titulado como Nueva Izquierda dirigido por José Murat ("Lima") que pronto formaría el grupo "Baluarte". (Ver http://eltopoblindado.com/files/Publicaciones/Organizaciones\%20Politico-

Militares\%20de\%20origen\%20Marxista/B\%20Baluarte/Prensa/N\%204\%20diciembre\%201963.pdf).

Encontramos varias veces el uso de "Nueva Izquierda" en Murat, quien ubicará dentro de la "vieja izquierda" tanto a Codovilla y Ghioldi, pero también a Silvio Frondizi, Jorge Abelardo Ramos, David Tieffenberg, entre otros referentes que los trabajos sobre el tema no dudan en referenciar como Nueva Izquierda. 2) Una revista dirigida por Horacio Daniel Rodríguez, proveniente del socialismo, donde lejos de asumir acercamientos o relecturas respecto del peronismo como toda la llamada Nueva Izquierda, se afirma que "el peronismo no existe" en tanto carece de la fuerza política que se le asigna, y que "continúa constituyendo una corriente afín con el fascismo" (Nueva Izquierda, № 4. Noviembre de 1963). Es decir que desde el punto de vista de los actores la categoría, las pocas veces que se utiliza, no tiene un significado cercano al uso analítico que se le ha dado, y se hace difícil sostener la existencia de un actor político unificado en torno a la llamada Nueva Izquierda.

${ }^{25}$ Terán no coloca entrecomillada a la categoría, en cambio Altamirano sí lo hace.

${ }^{26}$ ¿Qué es la izquierda? Cuadernos de Cultura Año XI / 50. Los textos de ese número son reproducidos en Giudice, E., et al, Buenos Aires, Editorial documentos, 1961. 
En definitiva, la categoría de nueva izquierda no fue asumida por los actores que son incluidos bajo esa denominación por los trabajos académicos. En ese sentido, para el caso argentino, resulta claramente una categoría conceptual y no "nativa". Pero más que descartar o aceptar la riqueza analítica de esa categoría, sugerimos problematizarla.

Algunos estudios sobre casos particulares han reconocido similares problemas a la hora de ubicar experiencias concretas dentro o fuera de la llamada nueva izquierda. A las tensiones ya mencionadas en diversos trabajos en torno a la ubicación de la izquierda peronista, hay que añadir otras. Hilb ya había señalado las "grandes similitudes en la concepción con que los grupos de la $\mathrm{NI}$ (...) conciben los sucesos que se desarrollan en el país (...) a excepción del PO y del PST”, por lo que agrega: “(De ahora en más, nos referiremos a la NI excluyendo implícitamente al PO y al PST)” (HILB; LUTZKY, 1984, p. 23).

También Eduardo Weisz (2004) ha señalado las dificultades que entraña la dicotomía izquierda tradicional / nueva izquierda para un estudio de las guerrillas de origen trotskista, especialmente el PRT-ERP.

Similares tensiones encuentra Horacio Tarcus en torno al "marxismo olvidado" de Silvio Frondizi y Milciades Peña, a quienes prefiere ubicar en un "lugar intermedio y equidistante entre la izquierda tradicional y la nueva izquierda" (1996, p. 28).

No puede menos que llamar la atención la tan reiterada dificultad en torno a la ubicación de experiencias tan diversas: tanto el caso de las organizaciones peronistas, hayan o no adoptado la lucha armada como método, como el de la segunda guerrilla en importancia, como fue el ERP, y el de dos intelectuales que en otros casos han sido ubicados como claros referentes de una nueva izquierda intelectual. ${ }^{27}$

Como se ve, diversos estudios concluyen que la categoría de nueva izquierda, conjunto heterogéneo de espacios políticos e intelectuales, no resulta tan útil para su objeto particular. Tal vez es más prudente preguntarnos — como lo hacía Kant— si no es el punto de vista del espectador (del investigador) el que debe ser problematizado cuando no consigue explicar aquello que observa. Podría afirmarse que estamos ante una

\footnotetext{
${ }^{27}$ Lo cual es especialmente válido en el caso de Silvio Frondizi, considerado un fundador de esa Nueva Izquierda por su protagonismo en la formación del grupo Praxis (AMARAL, 2005; TARCUS, 2007).
} 
categoría heredada cuya genealogía no siempre es tenida en cuenta y cuya utilización ha llevado a incrementar la lista de espacios que los investigadores ubican como casos excepcionales, ambiguos o equidistantes. Al no cuadrar su objeto en una categorización establecida con pretensiones generalizadoras, se le adjudica estar afectado por una variable no prevista aplicando sin decirlo la famosa cláusula ceteris paribus. ${ }^{28}$

\section{A modo de cierre}

Se puede concluir que la categoría de nueva izquierda, de uso tan extendido en las investigaciones actuales sobre la historia reciente, no se corresponde en extensión con la perspectiva que los propios actores le dieron a sus acciones políticas. Su uso en Argentina surge de la extrapolación de experiencias exógenas mediadas por una serie de trabajos canónicos sobre el tema que a la vez sirven como antecedente insoslayable para los trabajos más actuales.

Pero como categoría analítica aplicable al caso argentino, requiere ser definida de modo tal que las experiencias concretas puedan ser ubicadas dentro o fuera de esa delimitación, o si se opta por entrecomillar aludiendo a una "llamada «nueva izquierda»", habrá que definir entonces quién la llama así y por qué. Sostenemos que en caso de utilizar la categoría sería más prudente ubicar sin reservas a la izquierda peronista como uno de sus componentes centrales. Del mismo modo en que en otras latitudes se articularon marxismo y tradiciones políticas laboristas (Inglaterra), nacional-populares o populistas (México) y anticolonialistas (Francia-Argelia) (HALL, 2010), puede establecerse que en nuestro país fue significativa la articulación de elementos deudores de una tradición marxista en proceso de revisión con espacios políticos y figuras intelectuales que se identificaron como peronistas. Tal fue una de las principales particularidades que aquel fenómeno trasnacional adquirió en nuestro país, dando lugar a un entramado heterogéneo de espacios políticos e intelectuales durante las décadas del sesenta y

\footnotetext{
${ }^{28}$ La cláusula ceteris paribus consiste en incorporar una variable que modifica la relación establecida entre los fenómenos. Su uso hace del fenómeno estudiado un caso de excepción o que no cumple la regla por no estar dadas todas las condiciones. Puede aceptarse la afirmación de que dicha cláusula "no resulta necesaria si se especifican todas las condiciones en que se supone que tiene lugar algo" Ferrater Mora, José, “Ceteris Paribus”, en Diccionario de Filosofía, Barcelona, Ariel, 2004, tomo 1, p. 528.
} 
setenta que nominamos analíticamente como "izquierda peronista” y que los actores Ilamaron "tendencia revolucionaria del peronismo", "peronismo revolucionario", "nacionalismo popular revolucionario", entre otras denominaciones.

Pero más allá de esas consideraciones analíticas, la llamada nueva izquierda debe ser considerada como categoría utilizada por los actores en un contexto muy particular. Sus primeros usos se remontan, como vimos, a la utilización de diversos espacios políticos que buscaron contraponerse a la izquierda tradicional europea, en un contexto mundial de revisión, no solo del marxismo, sino de todas las herencias. Dicho proceso que comenzó a mediados de los años cincuenta llegó a su más alta expresión en los movimientos del '68. Se trató también de un período de ruptura generacional, de un fuerte rechazo por parte de los jóvenes al mundo que sus padres ofrecían, fenómeno que merece un estudio en sí mismo. Pero cabría preguntarnos en qué medida la idea de una nueva izquierda no se insertaba en un conjunto más amplio de significaciones en lo que todo “lo viejo" parecía ponerse en cuestión. Así, la idea acuñada por Ernesto Guevara de la construcción del "hombre nuevo", la idea de una "nueva izquierda", o la de una "nueva universidad", fenómeno que hemos analizado (FRIEDEMANN, 2016), se enmarcaban todas en un mismo proceso político y cultural a escala global de cuestionamiento a lo establecido. Tal vez, la dificultad a la hora de ubicar experiencias particulares dentro de la distinción realizada entre nueva izquierda e izquierda tradicional estriba en que más allá del uso que los actores hicieron de la dicotomía entre lo nuevo y lo viejo, el resultado social de esas manifestaciones epocales no podía más que estar basado en una configuración que contuviera elementos de ambos extremos de la díada. 


\section{Referências}

ACHA, O. La nación futura: Rodolfo Puiggrós en las encrucijadas argentinas del siglo XX. Buenos Aires: Eudeba, 2006.

ACHA, O. Historia crítica de la historiografía argentina: las izquierdas en el siglo XX. Buenos Aires: Prometeo, 2009.

ALTAMIRANO, C. Peronismo y cultura de izquierda (1955-1965). College Park, Latin American Studies Center, University of Maryland, n. 6, 1992.

AMARAL, S. Silvio Frondizi y el surgimiento de la nueva izquierda. Documentos de Trabajo, n.313. Buenos Aires: Universidad del CEMA., 2005.

BARLETTA, A. M. Universidad y política:la peronización de los universitarios. LASA Proceedings, 2000.

BARLETTA, A. M.; LENCI, M. L. Politización de las Ciencias Sociales en la Argentina. Incidencia de la revista Antropología zer. Mundo. Sociohistórica, v. 8, 2000.

BURGOS, R. Los gramscianos argentinos: cultura y política en la experiencia de Pasado y Presente. Buenos Aires: Siglo XXI, 2004.

CAMPOS, E. Cristianismo y revolución: El origen de Montoneros: violencia, política y religión en los 60. Buenos Aires: Edhasa, 2016.

CARLI, S. El viaje de conocimiento en las humanidades y las ciencias sociales. Un estudio de caso sobre profesores universitarios en la Argentina durante la segunda mitad del siglo XX. Historia de la Educación, Anuario, v. 2, n. 14, 2013.

CELENTANO, A. Insurrección obrera y compromiso intelectual. Los libros y Cristianismo y Revolución frente al Cordobazo y el Viborazo. Archivos de historia del movimiento obrero y la izquierda, v. 2, n. 4, 2014.

CHAMA, M. Peronización y radicalización de grupos de abogados en los años sesenta y principios de los setenta. La labor defensista como práctica militante. Cuestiones de Sociología: Revista de Estudios Sociales, n. 3, p. 143-168, 2006.

DE AMÉZOLA, G. El caso del realismo insuficiente. Lanusse, la Hora del Pueblo y el Gran Acuerdo Nacional. En: PUCCIARELI, A. (Ed.). La primacía de la política: Lanusse, Perón y la Nueva Izquierda en tiempos del GAN. Buenos Aires: Eudeba, 1999. p. 57-116. 
DE RIZ, L. La política en suspenso, 1966-1976. Buenos Aires: Paidós, 2000.

DIP, N. A. Libros y alpargatas:las tramas discursivas y organizativas del proceso de peronización de estudiantes, docentes e intelectuales de la Universidad de Buenos Aires. 1966-1974. [s.l.]: Universidad Nacional de La Plata, 2017.

DONATELLO, L. M. Catolicismo y Montoneros: religión, política y desencanto. Buenos Aires: Manantial, 2010.

FRIEDEMANN, S. El marxismo peronista de Rodolfo Puiggrós: una aproximación a la izquierda nacional. Buenos Aires: Instituto de Investigaciones Gino Germani, 2014.

FRIEDEMANN, S. La Universidad Nacional y Popular de Buenos Aires (1973-1974): una reforma universitaria inconclusa.2016. Tesis doctoral en Ciencias Sociales. Universidad de Buenos Aires. Facultad de Ciencias Sociales, 2016.

FRIEDEMANN, S. La peronización de los universitarios como categoría nativa (1966-1973). Folia Histórica del Nordeste, n. 29, 2017.

GEORGIEFF, G. Nación y revolución: itinerarios de una controversia en Argentina (19601970). Buenos Aires: Prometeo, 2009.

GILMAN, C. Entre la pluma y el fusil: debates y dilemas del escritor revolucionario en América Latina. Buenos Aires: Siglo XXI, 2012.

GÓMEZ, S. El derrotero de Los Libros (1969 - 1976) y su crítica pedagógica. La interpretación educativa de Antonio Gramsci a través de Christine Buci-Glucksmann. Izquierdas, n. 28, p. 292-314, 2016.

GONZÁLEZ CANOSA, M. ¿Democracia y/o Revolución? Las Fuerzas Armadas Revolucionarias frente a la coyuntura electoral: los comicios, la revolución y la lógica instrumental (Argentina, 1972-1973). Izquierdas, n. 38, 2018.

HALL, S. Vida y momentos de la primera Nueva Izquierda. New Left Review (español), n. 61, p. 163-182, 2010.

HERRERA, C. M. ¿Adiós al proletariado? El Partido Socialista bajo el peronismo (19451955). Buenos Aires: Imago Mundiu, 2016.

HILB, C.; LUTZKY, D. La nueva izquierda argentina: 1960-1980. [s.I.] Centro Editor de América Latina, 1984. v. 70 
HOBSBAWM, E. La influencia del marxismo. 1945-1983. En: Cómo cambiar el mundo.

Buenos Aires: Crítica, 2013.

KOHAN, N. En la selva: (Los estudios desconocidos del Che Guevara. A propósito de sus Cuadernos de lectura de Bolivia). Montevideo: Amauta insurgente, 2011.

MANZANO, V. Argentina Tercer Mundo: Nueva Izquierda, emociones y política revolucionaria en las décadas de 1960 y 1970. Desarrollo Económico, p. 79-104, 2014.

MORELLO, G. El Concilio Vaticano II y la radicalización de los católicos. En: LIDA, C.; CRESPO, H.; YANKELEVICH, P. (Eds.). Argentina 1976: estudios en torno al golpe de Estado. México: FCE, 2007. p. 111-130.

PONZA, P. Existencialismo y marxismo humanista en los intelectuales argentinos de los sesenta. Nuevo Mundo Mundos Nuevos: Nouveaux mondes mondes nouveaux-Novo Mundo Mundos Novos-New world New worlds, Local:Editora, 2006.

PUCCIARELLI, A. R. La primacía de la política: Lanusse, Perón y la Nueva Izquierda en tiempos del GAN. [s.l.] Eudeba, 1999.

REVEL, J. Un momento historiográfico: trece ensayos de historia social. Buenos Aires: Manantial, 2005.

SIGAL, S. Intelectuales y poder en la década del sesenta. Buenos Aires: Puntosur, 1991.

SIGAL, S.; TERÁN, O. Los intelectuales frente a la política. Punto de Vista, n. 42, 1992.

TARCUS, H. El marxismo olvidado en la Argentina: Silvio Frondizi y Milcíades Peña. Buenos Aires: El cielo por asalto, 1996.

TARCUS, H. Diccionario biográfico de la izquierda argentina de los anarquistas a la \&quot;nueva izquierda\&quot: 1870-1976. [s.l.] Emecé, 2007.

TARCUS, H. Marx en la Argentina:sus primeros lectores obreros, intelectuales y científicos. Buenos Aires: Siglo XXI, 2013.

TERÁN, O. Nuestros años sesentas: la formacion de la nueva izquerda intelectual en la Argentina 1956-1966. Buenos Aires: Puntosur Editores, 1991.

THOMPSON, E. Out of Apathy. London: New Left Books, 1960.

TORTTI, M. C. Protesta social y ‘nueva izquierda’ en la Argentina del Gran Acuerdo 
Nacional. En: La primacía de la política: lanusse, Perón y la Nueva Izquierda en los tiempos del GAN. [s.I: s.n.]. p. 205-231.

TORTTI, M. C. La Nueva Izquierda a Principios de los' 60: Socialistas y Comunistas en la Revista CHE. Estudios sociales, v. 22, n. 1, p. 145-162, 2002.

TORTTI, M. C. El viejo partido socialista y los orígenes de la nueva izquierda. [s.l.] Universidad Nacional de La Plata. Facultad de Humanidades y Ciencias de la Educación, 2007.

WEISZ, E. EI PRT-ERP: Nueva izquierda e izquierda tradicional. Buenos aires: Centro Cultural de la Cooperación, 2004.

ZOLOV, E. Expandiendo nuestros horizontes conceptuales: El pasaje de una «vieja» a una «nueva izquierda» en América Latina en los años sesenta. Aletheia: Revista de la Maestría en Historia y Memoria de la FaHCE, v. 2, n. 4, 2012. 